\title{
LA CONSTRUCCIÓN DE LO PÚBLICO MEDIADO POR UN DISPOSITIVO HIPERMEDIAL DINÁMICO
}

\author{
Patricia S. San Martín (CIFASIS- CONICET- UNR-UPCAM)* \\ Silvana Martino (CIFASIS- CONICET- UNR-UPCAM)**
}

\begin{abstract}
Resumen
El artículo presenta la problemática de diseño y desarrollo de un proyecto educativo transversal implementado en el contexto físico-virtual de una localidad de la provincia de Santa Fe, Argentina, cuya finalidad se centra en posibilitar nuevas formas de participación responsable de la ciudadanía para la construcción de lo público integrando las Tecnologías de la Información y Comunicación. El enfoque adoptado conceptualiza dicha construcción no restringida únicamente al espacio de gobierno, sino en la apropiación de éste por parte de la ciudadanía como estrategia de gobernanza, proceso a partir del cual es posible generar redes sociotécnicas que permitan el abordaje de problemáticas locales y la puesta en obra de distintas propuestas para su resolución. Se refieren los avances de la investigación para la configuración de un Dispositivo Hipermedial Dinámico que desde las instituciones educativas de nivel medio y la Secretaria de Cultura de la localidad, posibilite la escritura colaborativa de un libro "online" de las memorias colectivas por parte de los ciudadanos vinculando el hoy con la memoria del ayer en función del diseño e implementación de Políticas que permitan la articulación entre lo singular y lo colectivo y la responsabilización en la construcción de lo público como espacio integrador.
\end{abstract}

\section{Palabras clave}

Lo público - TIC - Participación responsable - Ciudadanía - Memoria colectiva.

Doctora en Humanidades y Artes. Universidad Nacional de Rosario. Profesora Nacional de Música. Facultad de Humanidades y Artes. Universidad Nacional de Rosario.

* Doctora en Humanidades y Artes. Mención Antropología. Facultad de Humanidades y Artes. Universidad Nacional de Rosario. Magister en Sociología y Ciencias Políticas. Facultad Latinoamericana de Ciencias Sociales. Becaria Pos Doctoral CONICET- CIFASIS. 


\section{Summary}

The article presents the problems of design and development of an educational project implemented in the physical-virtual context of a village in the province of Santa Fe, Argentina, whose purpose focuses on the possibility of new forms of responsible citizen participation to build of the public by integrating Information Technology and Communication. The approach conceptualizes the construction is not confined solely to the area of government, but ownership of it by citizenship and governance strategy, a process from which it is possible to generate socio-technical networks that allow the approach to local issues and making in the work of various proposals for resolution. They concern the progress of research for the configuration of a Dynamic Hypermedia Device from middle-level educational institutions and the Cultural Center of the locality, enable collaborative writing of a book "online" from the collective memories by linking citizens the presents with yesterday remembrans in terms of design and implementation of policies designed to articulate the singular and the collective and responsibility in the construction of what is public.

\section{Key words}

The public - ICT - Responsible participation - Citizens - Collective memories.

\section{Introducción}

Las dinámicas Tecnologías de la Información y la Comunicación (TIC) han producido profundas modificaciones en la sociedad del siglo XXI, habilitándose nuevos canales para el desarrollo social y productivo que posibilitan reflexionar sobre otras formas de construcción y despliegue de políticas públicas participativas en el actual contexto físico-virtual. Sin embargo, es observable el diferencial tecnológico que existe a nivel global generando asimetrías excluyentes en aquellas comunidades que quedan por fuera del uso significativo y potenciador del acceso a la información y el conocimiento, por múltiples causas vinculadas a condiciones socioculturales, económicas, generacionales y de accesibilidad entre otras.

La necesidad de construcción de un contexto físico-virtual inclusivo a nivel regional, instala debates sobre sus efectos en los procesos organizacionales y productivos, atendiendo a modalidades de interacción comunicacional, calidad y tipos de mediaciones y mediatizaciones, disponibilidad de infraestructura TIC y de servicios, adaptabilidad, flexibilidad y dinamismo de los sistemas digitales en uso, atención a la diversidad cultural, metodologías de trabajo individual y colectivo para la apropiación, producción y diseminación abierta del conocimiento a través de redes sociotécnicas colaborativas y de participación ciudadana para la calidad de vida, problemáticas que se constituyen centrales en las agendas de investigación, desarrollo e innovación de Argentina. 
La integración de las TIC en el campo educativo, de gobierno, de investigación y producción como así también en su uso social comunitario, solicitan de una propuesta contextualizada a la realidad local y a la singularidad de los ciudadanos para el despliegue de una participación activa y plural en políticas públicas centradas en la "salud de vida". Las instituciones se reconfiguran a partir de este nuevo contexto físico-virtual y los cambios más profundos no dependen únicamente de disponer de los insumos tecnológicos y la conectividad a internet, sino de las apropiaciones que cada sujeto en relación con el otro pueda realizar.

En este sentido, el presente trabajo referirá los avances del proyecto de I+D: "Wheelwright, comuna físico virtual: Diseño e implementación de un Dispositivo Hipermedial Dinámico para la construcción de civitas" seleccionado en la convocatoria INNOVA 2009 de la Secretaría de Estado, Ciencia, Tecnología e Innovación de la provincia de Santa Fe, Argentina. El mismo, es parte del Proyecto "Obra Abierta: DHD para educar e investigar" del Programa de Investigación, Desarrollo y Transferencia "Dispositivos Hipermediales Dinámicos", radicado en el CIFASIS (CONICET-UNR-UPCAM).

La metodología de trabajo se enfoca en cómo desarrollar procesos de participación y apropiación del espacio público físico-virtual mediatizado por herramientas tecnológicas por parte de todos los ciudadanos de una comunidad en particular. Procesos en el caso planteado, que por su misma dinámica, devienen en conflicto, consensos y acuerdos acerca de los modos, accesos, y significaciones en la producción de las memorias colectivas mediadas por un Dispositivo Hipermedial Dinámico. La estrategia puesta en juego, propone llevar adelante la escritura abierta y colabortiva de un objeto dinámico físicovirtual complejo, al que hemos denominado "Telares de la memoria", concebido en su diseño, desarrollo e implementación como un proceso de aprendizaje emergente hacia la producción de "civitas" (Laddaga, 2010).

Adquieren entonces especial énfasis, aquellas categorías ejes que atravesarán tanto la reflexión como las prácticas de esta propuesta, que si bien se sitúan en los procesos locales de una práctica educativa y/o política singular, no dejan de estar anclados en nociones más generales, como ciudadanía, participación ciudadana que en cuanto práctica política se orienta a la toma de decisiones relacionadas al bien común, la gestión de lo publico, y las responsabilidades en términos de gobernanza, las cuales irán interpelando reflexivamente la práctica concreta de esta experiencia.

"Wheelwright comuna físico-virtual..." es un proyecto interdisciplinario que se sitúa en una localidad santafecina de 7000 habitantes, donde se realiza una propuesta de intervención concreta, centrada en la construcción de un Dispositivo Hipermedial Dinámico (DHD) que desde las instituciones escolares de nivel medio y de gobierno local (Secretaría de Cultura), despliega la posibilidad de escritura hipermedial abierta de la memoria colectiva plural por 
parte de todos los ciudadanos que habitan o habitaron en algún momento la comuna. Uno de los objetivos que guía la propuesta, es que la propia comunidad educativa formal y no formal pueda desarrollar líneas de acción hacia la factibilidad y sustentabilidad del DHD, vinculando el hoy con la memoria del ayer, integrando significativamente las TIC. La finalidad última se centra en que los ciudadanos de la localidad puedan sentirse capaces y partícipes del diseño y puesta en obra de Políticas que permitan la articulación entre lo singular y lo colectivo y la responsabilización en la construcción de lo público físico-virtual como espacio integrador.

Lo propuesto a su vez, se inscribe en necesidades de desarrollo social y productivo detectadas en el país donde es fundamental fortalecer tanto asociaciones democráticas extensas como la apropiación responsable de las TIC por parte de la ciudadanía en su conjunto, siendo ambos aspectos indisociables en el actual contexto global y una prioridad en el ámbito de las políticas públicas de Argentina.

A continuación, se referirán aspectos significativos del marco teórico y metodológico del proyecto, luego los avances efectuados hasta noviembre de 2010 sobre la construcción social y tecnológica del DHD experimental actualmente en desarrollo. Finalmente se expondrán breves reflexiones conclusivas sobre lo realizado y sus posibilidades de transferencia a otras comunidades de nuestro país.

\section{Construir un DHD en Wheelwright}

Se conceptualiza al DHD, como una red heterogénea (Foucault, 1991), conformada por la conjunción de tecnologías y aspectos sociales que posibilita a los sujetos en un nuevo contexto físico-virtual presencial, realizar acciones participativas de interacción responsable con el otro para aprender, dialogar, proponer, informar, diseñar, confrontar, investigar, componer, evaluar, diseminar, bajo la modalidad de taller físico-virtual, utilizando la potencialidad comunicacional, transformadora y abierta de lo hipermedial, regulados según el caso, por una "coordinación de contratos" (San Martín, Sartorio, Guarnieri y Rodriguez, 2008).

En este sentido, la composición abierta de "Telares de la memoria" como proceso y producto del proyecto "Wheelwright comuna físico-virtual: un DHD para..." procura la conformación activa de una red sociotécnica de participación responsable que posibilite tensionar los conceptos de participación y construcción de lo público en proyectos locales concretos, no necesariamente propuestos por el gobierno local, habilitando la comprensión de los sentidos, significaciones e intereses que se elaboran en torno a la producción de "civitas".

En la revisión de una amplia bibliografía sobre la escritura de la memoria, se desprende que los eventos acaecidos son un dato pero el pasado no es nunca un dato en sí, fijo e inmutable porque la memoria de los hechos en cuanto 
eventos dados es absolutamente variable y renegociable y, el acto de constante re-escritura del pasado está siempre anclado en el presente. Entonces, toda construcción colectiva, y en este caso, el de la memoria, implica juegos de intereses, conflictos, y negociaciones en tanto que la memoria es objeto de una lucha en el presente en la que ciertos grupos intentan apropiarse de ella. Se interpreta, tal como lo expresa Nora (1997), que la memoria, por naturaleza, es afectiva, emotiva, abierta a todas las transformaciones, inconscientes de sus sucesivas transformaciones, vulnerables a toda manipulación, susceptible de permanecer latente durante largos periodos y de bruscos despertares. La memoria es siempre un fenómeno colectivo, aunque sea psicológicamente vivida como individual. Coincidimos con Violi (2010), cuando refiere la necesidad de construcción de la memoria, como una memoria potencialmente "compartible": una perspectiva asumida y asumible por una sociedad dada que funda un modo común de mirar su propio pasado y de proyectar su futuro, de allí lo colectivo.

Entonces, la construcción de la memoria en su sentido plural, se entrama a las nociones de lo político, en sus usos y manipulaciones, a partir de lo cual es posible poner en juego construcciones de lo público, de lo colectivo. Así, en "Telares de la memoria", las instituciones/organizaciones involucradas y su amplia comunidad, podrían desplegar significativamente su "presencia" a partir de estrategias de producción, inclusión y uso de lo público centradas en la posibilidad de crear "civita", otorgando sentido pleno a la ciudad "físico-virtual" como lugar productor de ciudadanía y ámbito del ejercicio de ésta.

A partir de la interrogación sobre cómo se retroalimentan significativamente procesos de gestión política comunales, desde una experiencia de participación comunitaria proyectual que integre herramientas TIC, se analizaron diversas propuestas a nivel regional, nacional e internacional de las actuales políticas culturales diseñadas como espacios físico-virtuales de gobierno participativo e inclusivo, tendientes a la construcción de ciudadanía. Además, se estudiaron, no sólo las propuestas generadas y propiciadas desde el ámbito gubernamental, sino también aquellas de base participativa que integran TIC, construidas en esferas públicas no gubernamentales.

Así, iniciativas como "Park Fiction", "Wu Ming", "La Comuna", "What's de time in Vyborg?" reseñadas y analizadas en profundidad por Laddaga (2010), se constituyen por su perfil en antecedentes significativos del presente caso, siendo proyectos que surgen de lo artístico a finales del siglo $X X$ integrando metodologías y tecnologías provenientes de distintas disciplinas dando cuenta de una construcción interdisciplinaria de producción dinámica y compleja por la heterogeneidad del dispositivo puesto en obra. En sus fundamentos, se sostienen básicamente a partir de la "producción colaborativa de deseos", la escritura sobre lo social abierta y comprometida, la discusión de una colectividad sobre sus problemas y la memoria plural como estrategia de construcción de presente y futuro. Proyectos todos, donde el patrimonio tangible e intangible cobra presencia y significación a partir de la voz de una amplia comunidad de 
"expertos y no expertos", jóvenes y adultos comprometidos y convocados por lo público, reunidos en la discusión y en el desarrollo de una mirada atravesada por el conflicto y la creatividad, asumiendo el rol de gestores y movilizadores de una comunidad cada vez más amplia e inclusiva.

Si bien en los últimos años, distintos gobiernos han apoyado acciones de integración de TIC en los campos educativos, de gobierno y salud y, se disponen actualmente de herramientas tecnológicas y sistemas informáticos que posibilitan la interactividad social, aún hay fuertes debilidades que muestran un profundo contrasentido conceptual y operativo con los fundamentos que posibilitaron la construcción de redes dinámicas colaborativas como Internet. Siguiendo a Thomas y Buch (2008), se postula que los procesos de producción, apropiación y uso de la tecnología se tornan relevantes socialmente, si se gesta desde un principio un vínculo participativo entre los ciudadanos y organizaciones involucradas, que se sostiene a lo largo de la cadena procesual.

En referencia a lo observado sobre diversos sitios web (portales gubernamentales) de localidades de Argentina, es notorio que aún no se han potenciado las relaciones entre los distintos espacios organizacionales físicos-virtuales; las relaciones de la red de espacios físico-virtuales de las organizaciones con los múltiples espacios físicos-virtuales que habita el ciudadano y la utilización de herramientas interactivas en función de la producción y participación ciudadana inclusiva.

En su mayoría, los casos que se denominan en general como portales de "ciudades digitales", atienden principalmente a la mera transmisión de información o simples acciones administrativas de gestión. Se manifiestan en su diseño, como un reemplazo sutil de formas tradicionales de acceso a información, paquetes de baja flexibilidad que replican generalmente modelos con muy bajo dinamismo y que en sus efectos no habilitan un compromiso comunitario responsable hacia el desarrollo de conocimiento innovador ni a la implementación participativa de políticas públicas centradas en el bien común.

Organizaciones de Argentina como el CIPPEC (Centro de Implementación de Políticas Públicas para la Equidad y Crecimiento) que se dedican a la temática en relación a la participación mediatizada, entienden que estas novedosas formas podrían provocar la constitución de nuevos paradigmas, por ejemplo en la participación y representación política mediadas por las nuevas TIC y diagnostican que en nuestro país el ciudadano medio informado no puede hoy identificar los "key issues" de la agenda política ni encontrar información resumida y balanceada para tomar una posición bien sustentada. Y aún cuando lo hiciera, no tendría claro de qué modo podría influir sobre el proceso político. Desde la perspectiva de las autoras de este trabajo, se sostiene que la participación no queda garantizada con el sólo uso de renovadas herramientas tecnológicas y el diseño de portales virtuales, si éstos aún siguen siendo diseñados de un modo unidireccional. El objeto tecnológico interactivo puede habilitar la participación pero no garantiza ni asegura el compromiso responsable. Si la información 
sigue apareciendo como estática, dada y "objetiva" es poco posible pensar en una participación con dimensión transformadora.

El marco teórico y metodológico del presente proyecto, refiere a la perspectiva de los "sistemas complejos" (García, 2007), lo cual implica poder conceptualizar la propuesta recortándola en la realidad compleja de la comunidad. Situarse en la instituciones públicas educativas formales y no formales y, comprender además, las interacciones entre los procesos que se desarrollaban en cada uno de sus subsistemas, solicita referenciarlos permanentemente a sus respectivas historias para posibilitar la puesta en obra de la noción "DHD Wheelwright" que excede lo meramente instrumental de lo tecnológico informático (San Martín, Guarnieri, Rodriguez, Bongiovani y Sartorio, 2010). Desde los aspectos sociales del dispositivo, lo expuesto significa abordar en el mediano y largo plazo, acciones de promoción en la ciudadanía que incentiven hacia el despliegue de políticas de prevención/seguridad/producción comunitaria.

El enfoque DHD, integra campos complementarios y sostiene que actualmente los cambios culturales relacionados a procesos de innovación y compromiso participativo en las prácticas, devienen más lentos que los desarrollos y operatividad de las tecnologías TIC involucradas. Entonces, se procura, que más allá de las capacitaciones realizadas en función del primer prototipo experimental de "Telares de la memoria", se construya paulatinamente y en profundidad una toma de conciencia activa hacia las problemáticas de la localidad a partir del crecimiento textual colaborativo del objeto virtual.

Estas problemáticas serán relevantes si de la discusión y planteamiento de contenidos, se construyen prácticas ciudadanas, en tanto que permitan la apropiación y resignificación entre todos los actores locales involucrados, para la búsqueda consensuada de alternativas de resolución a dichos problemas. Para esto, es necesario generar procesos que transformen la gestión de lo público atravesada por una visión unidireccional y univoca, restrictiva de la riqueza que brinda la coproducción multiagencial (tanto estatal como societal) que da consistencia a la cosa pública. (Borja, 2004, Crawford, 1998, Lea y Young, 2001, Young, 2003).

\section{2-1 Sostener la presencia.}

De lo expuesto, se sintetiza que construir un DHD, significa tanto en lo teórico como en lo metodológico, desarrollar una mirada relacional, polifónica e hipertextual (San Martín y Guarnieri, 2009) centrados en la inclusión social, el respeto a la diversidad, el acceso abierto a la información y el conocimiento, la libre participación ciudadana, reconceptualizando lo presencial más allá de la positividad física, como dimensión simbólica.

El problema relevante en una sociedad donde las TIC atraviesan la cotidianeidad pública y privada, interroga sobre cómo sostener la "presencia" subjetiva y la participación ciudadana responsable e inclusiva más allá del grado de mediatización. Así surge como estrategia primordial, hacer lugar a la multiplicidad de lo 
emergente a partir de una instancia común y abierta entretejida en este caso de memorias y olvidos (Candau, 2002), creando superposiciones y vínculos que otorguen nuevas dimensiones a los "hipertextos" y habiliten situaciones de aprendizaje a partir de la composición compleja del proceso físico-virtual colaborativo.

Cabe recordar que procesos compositivos abiertos basados en lo emergente, donde el público se constituye en coautor a partir de la improvisación (gestual, sonora, visual, etc.), vistos en variadas instalaciones y obras de distintos géneros y soportes, son entre otros, recursos y técnicas de larga data, que posibilitan a los participantes experiencias subjetivas significativas abriendo las puertas a diversos modos de aprendizaje y expresión (San Martín, 2003). Plantear la problemática de este modo, excede el tradicional dualismo presencial - a distancia y lo meramente cuantitativo del grado de mediatización, centrándose el debate en su real dimensión política y educativa dada la emergencia de nuevas formas culturales físico-virtuales de aprendizaje, gestión, transmisión, producción y acceso -o no- a la información y conocimiento. Esto involucra para el desarrollo de conocimiento en sus aspectos sociales y específicamente en lo educativo, la presencia de técnicas, tecnologías y proyectos con dinámicas colaborativas y, la extensiva utilización abierta de herramientas digitales aptas para múltiples propósitos.

Se trata de habitar una "red social físico-virtual" que trascienda tanto al discurso monolítico del tradicional "manual escolar" como a la generalizada hiper-instantaneidad resultante de los populares "libros de perfiles" que habitan Internet. En este marco, el despliegue del "DHD Wheelwright", se realiza a través de mediatizaciones y mediaciones abiertas sustentadas en la modalidad de taller donde se reflexiona sobre los propósitos que lo convocan. Así, se asume al DHD como espacio público integrador donde es posible el desarrollo de procesos de aprendizaje para la producción de "civitas". Lo cual, implica un posicionamiento sobre las "competencias" requeridas, como capacidades que se sintetizan en el sujeto como un "saber hacer - saber ser", que se construyen como un saber "ha-ser" ético (Cullen, 2004), asumiendo la diversidad y la complejidad del contexto físico-virtual, expresándose a través de articuladas coordenadas de acción colectiva.

El diseño del proceso de investigación (2010-2011), se realizó bajo un enfoque metodológico cualitativo, considerando de manera flexible tres fases. La primera, atiende a una aproximación diagnóstica de la comunidad (Hammersley y Atkinson, 1994), la segunda trata sobre la construcción social y tecnológica del DHD, que implica el desarrollo e implementación de un primer prototipo experimental, finalmente la tercera fase tiene por objetivo evaluar la apropiación de la propuesta por parte de la comunidad.

Sobre el grupo investigador, cabe mencionar que es interdisciplinario proveniente de diversas disciplinas tales como Antropología, Arquitectura, Artes, 
Ciencias de la Computación, de la Educación, Historia, Ingeniería, Psicología y Trabajo Social.

En referencia al desarrollo del Primer Prototipo Experimental (PPE), "Telares de la memoria" servirá como documento para optimizar a futuro la presente propuesta, brindando una sencilla comprensión para públicos no-expertos, practicidad en el uso y su sentido amplio. La documentación elaborada sobre esta experiencia, quedará a disponibilidad pública como bien intelectual común bajo licencia Creative Commons y código abierto.

\section{Avances del proyecto}

A continuación se referenciarán los procesos más significativos que se fueron construyendo y reflexionando a nivel grupal durante las dos primeras fases (10 meses de desarrollo proyectual) sobre un total de dos años dedicados al caso (2010-2011). Se exponen seguidamente algunas consideraciones, logros parciales y problemáticas visualizadas.

\section{3-1 Fase 1:}

En el inicio fue necesario para el grupo del CIFASIS, recorrer en extenso el "territorio" donde se buscaría arraigar las prácticas y las reflexiones de la propuesta proyectual. Fue así que se comenzó con la construcción de lazos y vínculos con la ciudadanía donde se fueron constituyendo referentes locales ampliando el grupo gestor inicial. Estos referentes "habilitadores", presentaron lugares significativos, personajes, narrativas e interrogantes que fueron otorgando contextura a la propuesta.

Las primeras entrevistas realizadas, se vincularon en primer lugar al ámbito educativo: directoras y maestras ya jubiladas de las escuelas primarias y secundarias del pueblo (maestras de todos). En estas entrevistas, en compañía de los referentes, se iban explicitando los objetivos, las ideas generales del proyecto, y quedaban instalados interrogantes acerca de la construcción del mismo, de las condiciones para llevarlo adelante, enlazando la propuesta con sus propios relatos, sus experiencias y recuerdos posibilitadores del encuentro con otros actores claves de la historia del pueblo.

A su vez, los relatos de estos actores claves dieron cuenta que sus prácticas superaban en mucho el rol asumido en la institución escolar o en otras organizaciones. Así, fueron emergiendo algunas cuestiones que ganaron lugar en las reflexiones acerca del pasado, en tanto historia del pueblo, como en las condiciones y posibilidades actuales de prácticas ciudadanas. En el relato de las maestras y directoras (mujeres en amplia mayoría), aparecieron nociones acerca de la educación, y prácticas educativas más amplias con un compromiso activo con el contexto social de la localidad, promoviendo por ejemplo, el movimiento familiar que se generó a partir del pedido de apertura de una escuela secundaria para el pueblo. 
Estos relatos, llenos de recuerdos y de afectos, se fueron complejizando con la aparición de nuevos personajes claves, como lo fue la figura de un cura, de otra directora, de un productor agropecario, que iban atando, vinculando lugares, recuerdos, construyendo obras. El ir acompañando estas experiencias recordadas, revividas permitió ampliar la mirada y los recorridos que habían partido desde las escuelas.

Así se sucedían los entrelazamientos entre por ejemplo, una directora actual de una de las escuelas medias y su protagonismo en la gestión de Wheelwright como "Capital de la música" (1991); historias narradas desde los festejos del día de la música hasta su organización como comisión (año 2000), con sus acuerdos, conflictos y contramarchas; desde la guardería comunal (1970) como un proyecto de las mujeres trabajadoras hasta la fábrica textil más grande del país radicada en el pueblo desde los años 60; de la llegada de los trabajadores golondrinas en cada ciclo del maíz hasta el asentamiento de familias enteras migrantes del norte del país buscando trabajo urbano (1960); desde la reducción de la fábrica en la década del 90 y los despidos masivo de sus trabajadores en su mayoría del pueblo, a los nuevos modos de trabajo familiar, autónomo, empobrecido (a partir del 2001), entre una gran cantidad de relatos.

En estos recorridos, se entrecruzaban personas, lugares, vivencias, luchas, fracasos, logros, pero sobre todo aparecían fuertes acciones y prácticas que ligaban, formaban un "nosotros", lo cual permitía comprender los procesos en los cuales se fue construyendo lo público, lo de todos, lo común. De este modo, se fue componiendo el mapa vincular del pueblo, mapa en el que se inscriben las primeras marcas cuando algunos relatos se superponían en el tiempo, se contradecían en los significados, se oponían a los de otros, marcas que dejan huella de esa construcción plural y común.

Procesos significativos que permitieron discutir al interior del grupo CIFASIS, los modos posibles de leer y comprender la diversidad y pluralidad de las narraciones, recuerdos y memorias, posibilitando proponer en referencia al campo de contenidos tres macro categorías, a saber:

Obra Abierta: entendiéndose toda aquella construcción individual o grupal donde la ciudadanía puede acceder y/o participar y en las cuales tienen lugar no sólo los hechos que se concretaron sino todo aquello que formó parte de proyectos, anhelos, creaciones artísticas, etc. que no habiendo logrado su institucionalización pueden dar cuenta de construcciones de lo público como vehiculizador de dichas acciones. Como ejemplos recogidos en las entrevistas realizadas cabe mencionar el proyecto de constitución del Museo escolar y comunal; el primer periódico del pueblo; las actividades llevadas adelante por directivos, maestras, que permitieron situar a la escuela como un espacio social de inclusión; las gestiones para el reconocimiento de la música como parte identitaria del pueblo, publicaciones históricas sobre la localidad, expresiones literarias. 
Espacios habitados: en tanto aquellos donde fue necesario plasmar modos de participación y ejercicio de la ciudadanía, como ejemplo pueden encontrarse espacios institucionales que cuentan con la historia de su gestión pero que aún hoy siguen redefiniéndose, tales como: el club Popular y el club Italo; las bibliotecas públicas; el museo de la escuela primaria y luego de la comuna; la zona donde se asentaron los migrantes del norte del país; la festividad del día de la Música; las escuelas asentadas en el pueblo; la estación ferrocarril, la cual forma parte de uno de los relatos de fundación del pueblo; las distintas construcciones acerca del espacio usado para las "tertulias", etc.

En estos lugares es posible encontrar superposiciones, resignificaciones de mismo espacio en cuanto por ejemplo, a los usos que se dieron de ese mismo lugar, lo cual enriquece y complejiza los relatos.

Constructores/as de civitas: aquellos que formaron parte del proceso de discusión, organización y decisión para la puesta en marcha de acciones que tendieron a la producción de ciudadanía. Son ciudadanos que con sus prácticas trascendieron lo individual, lo privado, los límites de su labor específica para constituir situaciones y hechos públicos, en tanto que involucraron a otros, y tienen o tuvieron como objetivo el bien común.

El análisis de las posibles intersecciones de las categorías propuestas se hace indispensable a la hora de establecer un mapeo de la información, diseñar la posible navegación, el perfil de las interfaces, seleccionar las herramientas informáticas adecuadas, efectuar el modelado del DHD entre otros aspectos no menos importantes.

Sobre el análisis de los datos obtenidos en las entrevistas y observaciones, llevamos adelante una primera sistematización de todo el material recogido en una cartografía para la búsqueda de vinculaciones y lazos significantes entre los relatos, decires, los objetos, fotografías y los espacios institucionales. Los ejes que se comenzaron a trazar dieron cuenta en principio de:

- Aquellos silencios en relación a hechos o situaciones que modificaron la cotidianeidad del pueblo, por ejemplo el cierre de una de las fábricas de confección textil más importante de la región y del país, asentada desde 1960 en la localidad. El cierre o reducción de su plantel de trabajadores en la década de los 90 produjo variaciones en las formas tradicionales de trabajo, siendo en muchos casos el inicio de nuevas relaciones laborales. A partir de entonces, se fueron abriendo talleres independientes de producción de prendas textiles, en las mismas casas de familias, apareciendo la producción familiar como nueva organización de la economía, lo que implicó renovadas formas de transmisión del oficio, o la migración de mano de obra a los pueblos lindantes.

- Divisiones territoriales y simbólicas revitalizadas a partir de la pertenencia a determinados espacios, instituciones, partido político, o familias que reproduce tensiones y fragmentaciones en la población: vivir de un lado 
u otro del pueblo (1); participar de algunas de las instituciones sociales y deportivas; haber asistido a una u otra escuela, sobre todo en el nivel primario; etc.

- Tensiones acerca de la autoridad, control y hegemonía en la construcción de la historia común. Esto permitió, sobre todo en el ámbito escolar, poner en discusión la pluralidad y heterogeneidad en la construcción de lo colectivo, por ejemplo, sobre la superposición y contradicción de visiones acerca de hechos significativos en la historia del pueblo.

- Relatos de acciones colectivas donde la participación plural fue lo fundante en la construcción de identidad del pueblo. Son algunos de ellos, las fiestas de la música que se organizaron por iniciativa de algunos vecinos y una gestión política de gobierno alrededor de 1991 y la trascendencia que ese evento fue adquiriendo a partir de constituirse en un punto de encuentro e identidad en el pueblo como capital provincial de la música. Otras acciones las encontramos en la movilización de los ciudadanos para la apertura de la escuela secundaria en el pueblo, en el cierre de los comercios para llevar a cabo una protesta frente a elecciones "fraudulentas" en 1942, lográndose que triunfe la "voluntad ciudadana", etc.

Esta fase, si bien estuvo centrada en la posibilidad de construir una cartografía densa de vínculos y relaciones, permitió reconocer las distintas visiones que sobre estos territorios se construyen, algunas anudadas a la condición del actor que lo relata, que lo dibuja y lo describe (Reguillo 2005). A partir del cruzamiento del material relevado, se comenzó el diseño de "Telares de la memoria", como una trama posibilitadora del trazado de recorridos y trayectorias singulares donde cada sujeto desde un lugar contextuado pueda explorar la aparición de nudos o "lugares de la memoria" (Nora, 1997). Entonces, desde la diversidad de la textualidad generada por los diversos actores, pueden emerger algunos de estos nudos sentidos como punto de condensación entre lo vivido, lo recordado, lo significado, expresados con límites irregulares e inciertos (Halbwachs, 1994). Puntos de conflicto y desacuerdos podrían habilitar nuevas formas de significar.

Es en este sentido, que cobra fundamental importancia observar la calidad de los intercambios que hacen al proceso dinámico de desarrollo interactivo de la red sociotécnica que condiciona a su vez, la emergencia de formas de encuentro donde se sostenga un vínculo atento a otros decires y experiencias, necesario para seguir tejiendo tramas diferentes en el espacio pleno de un mismo telar. Cabe aclarar que dicho Telar en su diseño informático se constituye, dada su complejidad, de un conjunto de sistemas y herramientas web.

\section{3-2 Fase 2:}

Esta fase incluyó lo referido a los espacios de capacitación y lo inherente al desarrollo tecnológico del primer prototipo experimental. 
Sobre la organización de los espacios de capacitación físicos- virtuales en instituciones formales y no formales, se realizaron procesos de transferencia que implicaron el desarrollo de capacitaciones físico-virtuales en Escuelas Medias y Secretaria de Cultura (capacitación en servicio durante cinco meses).

Sobre la puesta en marcha de la segunda fase, si bien la propuesta original estaba centrada en la posibilidad de que fueran las escuelas secundarias las iniciadoras de la propuesta, en la primera fase se detectaron otros espacios donde era posible también su desarrollo. Uno de ellos, fue la Secretaria de Cultura que por su dependencia presentaba ambigüedades difíciles de resolver, como la de ser un lugar de mucho tránsito, aceptación y participación en el pueblo desde actividades concretas pero a su vez una referencia precisa anclada a la actual gestión de gobierno comunal. La problemática se fue diluyendo a través de fortalecer los vínculos entre las personas participantes en el hacer del propio proyecto lográndose que este espacio se pudiera habitar como un lugar donde es posible el encuentro de las diferentes voces en diálogo creativo. La Secretaría de Cultura, ubicada en los andenes de la estación de Wheelwright, fue entonces el espacio público del proyecto que funcionaría como contrapunto no formal al espacio escolar.

Seguidamente se diseñó e implementó, la capacitación en servicio para los docentes de las tres escuelas medias de la localidad y para los talleres de la Secretaria de Cultura de la comuna, los contenidos de la capacitación físico-virtual fueron diseñados en tres módulos: metodología de proyecto, marco teórico sobre participación ciudadana en relación al DHD y de operatoria en TIC. Se diagramaron encuentros en Wheelwright tanto en el ámbito escolar como en la Secretaria de cultura y variadas actividades en el espacio virtual de la capacitación. Se integró en un solo espacio virtual el área formal y el área no formal, promoviendo el intercambio entre docentes, alumnos y ciudadanos comprometidos en tareas concretas de producción del libro online.

Las capacitaciones a cargo del grupo CIFASIS, comenzaron en junio/julio del 2010, con la participación en la institución escolar de 45 docentes y directivos y 200 alumnos/as de los últimos años de las tre Escuelas de Enseñanza Media, las cuales comparten el mismo edificio. En el Centro de Cultura participaron en la capacitación 25 ciudadanos/as entre 16 y 80 años.

Los grupos asistieron en la primera jornada, a una introducción básica del contenido de los tres módulos a través de una dinámica activa de trabajo dando cuenta de la modalidad pedagógica de taller físico-virtual. La actividad realizada abordó tres interrogaciones básicas sobre la comprensión conceptual acerca de qué se entiende por "Prototipo experimental", "Participación responsable en contextos físicos-virtuales" y "Entornos virtuales colaborativos".

En los sucesivos encuentros se fue profundizando la transferencia a la comunidad educativa formal y no formal de herramientas teóricas, metodológicas y tecnológicas para la composición del prototipo de "Telares..." donde se escribirían pluralmente los relatos que hacen a la memoria colectiva de Wheelwright. 
La capacitación realizada tomó centralmente en cuenta el momento particular de este año 2010, donde confluyen las celebraciones por el bicentenario de Argentina y localmente en el mes de junio, los actos conmemorativos del cincuentenario de una de las escuelas participante, hecho que permite entramar con total pertinencia el proyecto propuesto.

Se destacan en este trayecto los siguientes avances y resultados parciales:

- Se diseñaron y efectuaron reuniones y encuentros presenciales en Wheelwright con un significativo número de participantes en los diferentes grupos de las distintas instituciones que integran el proyecto (área de educación no formal y formal) con el fin de poner en discusión los avances alcanzados del proyecto y brindar nuevas herramientas teóricas- metodológicas para la prosecución del libro.

- Los encuentros taller fueron también motivadores para discutir sobre el modo de llevar adelante el trabajo de campo que demandaría entre otras cosas la transferencia a la comunidad en general de las herramientas tecnológicas propuestas. Cabe destacar que fueron a la vez muy importantes las conversaciones en profundidad realizadas sobre los temas más significativos a partir de los cuales era necesario trabajar en la construcción de la memoria en torno a las categorías antes delineadas. La conformación de equipos de trabajo alrededor de estos temas y las discusiones acerca de los modos de recopilación, escucha y convocatoria a participar de aquellos que podían narrar, recordar y resignificar, provocaron que el grupo de trabajo palatinamente se fuera ampliando. En la actualidad, no sólo participan aquellos referentes locales que desde el comienzo habían guiado los primeros pasos, sino que se interviene desde distintos grupos temáticos y espacios diferenciados.

- La distancia que en un primer momento aparecía de acuerdo al lugar de anclaje del proyecto (escuelas secundarias- secretaria de cultura), empezó a esfumarse al constituirse grupos de trabajo donde no primó la relación institucional de quienes participaban sino más bien el interés y el conocimiento que el tema les había provocado.

- Las dimensiones (constructores, obras, espacios habitados) que, puestas en relación, constituyen el núcleo de "Telares..." y que propician la participación desde las distintas visiones acerca de la memoria, resultaron efectivas para el ordenamiento de las actividades y la conceptualización de la propuesta por parte de la comunidad local.

- Es de destacar el compromiso asumido en esta fase del proyecto por la comuna local, facilitando sin ningún tipo de inconveniente las herramientas y conexiones a internet en cada uno de los espacios donde se asentó el proyecto, como así también la disponibilidad hacia el proyecto, de la comisión que preside actualmente dicha institución. 
- Se brindó tutoría en la localidad y tutoría virtual tal como se había diseñado, utilizando en un primer momento el espacio virtual habilitado ad hoc de "Comunidades" del Campus Virtual de la Universidad Nacional de Rosario (UNR) (www.campusvirtualunr.edu.ar), cumpliendo a su vez la doble función de acercar a los ciudadanos a la UNR, como una necesidad detectada en el prediagnóstico efectuado durante el 2009.

- El proceso expuesto no estuvo exento de conflictos de poder, recelo hacia la propuesta, a su vez posibilitó la emergencia de fragmentaciones políticas e institucionales, lo que motivó a algunos a no participar o a establecer ciertas condiciones de participación, siendo un desafío constante poder discutir la necesariedad de que sea un espacio donde todos puedan contar, discutir, aportar, hablar. Sin embargo este punto de conflicto es el más importante que da sentido a la propuesta y que motiva la posibilidad de seguir llevándola adelante.

\section{3-3 Desarrollo del Primer Prototipo experimental}

Continuando con la fase 2, seguidamente se exponen los avances de las actividades relacionadas con la construcción social y tecnológica del Prototipo Experimental de "Telares de la memoria" que se constituyó a partir de la organización de grupos transversales (instituciones de educación formal y no formal) en torno a temáticas y contenidos del libro on line.

Sobre lo social, a partir de la sistematización y primeros análisis de los datos obtenidos en la Fase 1, como ya se expuso, se ponderaron ejes temáticos significantes para los propios habitantes de la localidad. En entrevistas grupales y reuniones formales e informales cada eje fue constituyéndose en un espacio de co-gestión (en algunos casos comuna-secretaría de cultura-escuelas medias y ciudadanos; escuela-docentes y alumnos; ciudadanos y organizaciones de la sociedad civil-radios-clubes) para la discusión sobre las decisiones acerca del qué (contenidos de los ejes); la metodología más apropiada para recoger los datos necesarios; y a quiénes o con quiénes trabajar en esta etapa de construcción. Estos espacios grupales se sostuvieron de manera presencial y virtual, dinámica de trabajo propuesta para todo el proyecto.

La construcción social y tecnológica del DHD, se fue enriqueciendo en el proceso de capacitación mencionado, en cuanto a las visiones, representaciones de los propios ciudadanos de Wheelwright sostenida en la implementación de una primera interface original del prototipo que muestra un sistema de almacenamiento digital abierto de información tipo "repositorio". El mismo, da cuenta de las tres dimensiones mencionadas (Obras, constructores y espacios) y permite la subida, localización espacial y simple indexación en línea (atendiendo a estándares internacionales) de todo el material recogido en campo que los ciudadanos van aportando. 
Esta parte del DHD se ha configurado a modo de propuesta didáctica, reelaborando los aportes y desarrollos originales de Tosello y otros (2010), su desarrollo e implementación se centra en dos aspectos significativos. El primero, consiste en posibilitar a la ciudadanía aportante posteriores transferencias en temáticas referidas a las TIC, específicamente hacia una comprensión más integral (conceptual y operativa) de sistemas de almacenamiento indexado de información como los repositorios de publicaciones en Acceso Abierto de alto impacto internacional; el segundo está centrado a nivel de grupo de $I+D$, en avanzar hacia propuestas innovadoras que mejoren los desarrollos colaborativos internacionales de repositorios de código abierto. La interface del sistema resulta totalmente innovadora ya que muestra dinámicamente las tres categorías propuestas y permite enlaces a otros sistemas asociados.

El "repositorio digital de Objetos de la Memoria" posibilitó a los ciudadanos visualizar las categorías planteadas, los formatos de recursos (contenidos) a aportar y la metodología para la carga, todo a modo de prueba exploratoria. A nivel del grupo de I+D del CIFASIS, se procedió en primer lugar a seleccionar y especificar metadatos en función de las dimensiones tratadas y luego, se desarrollaron las correspondiente fichas digitales de edición abierta en línea (tipo aporte wiki) con su instructivo para que los usuarios puedan efectuar adecuadamente y sin dificultades, la subida de contenidos y asegurar así el logro de una efectiva vinculación a nivel de la base de datos del sistema.

Sobre los aspectos que hacen al desarrollo de la interfaz visual y demás aspectos técnicos del DHD, los mismos exceden los límites del presente trabajo, sólo para una mejor comprensión se puede observar en la figura 1, la interface en construcción dinámica actualmente en línea que aparece luego de una breve introducción, habilitando desde el menú otros enlaces: foros de discusión, sitio del Programa DHD y carga de contenidos. Cabe aclarar que se simulan tres dimensiones utilizando sólo código HTML, constituyéndose esto un aporte y esfuerzo de programación adecuado a las bajas velocidades de transferencia que se registran en la localidad, similares a la mayoría de las localidades rurales de Argentina.

En síntesis, el esquema organizacional del espacio virtual de "Telares de la memoria", se configura entonces, con una introducción que conduce a una primera interface de entrada general que comunica las tres macro-categorías y habilita distintas posibilidades y posibles grados diferenciados de participación que delimitan a su vez, posibilidades de edición de contenido y desarrollo de actividades interactivas. De acuerdo a los intereses, perfiles y responsabilidades distribuidas de los ciudadanos participantes se podrían generar las siguientes situaciones de interactividad creciente y generación de simultaneidades utilizando las actuales herramientas colaborativas web: 


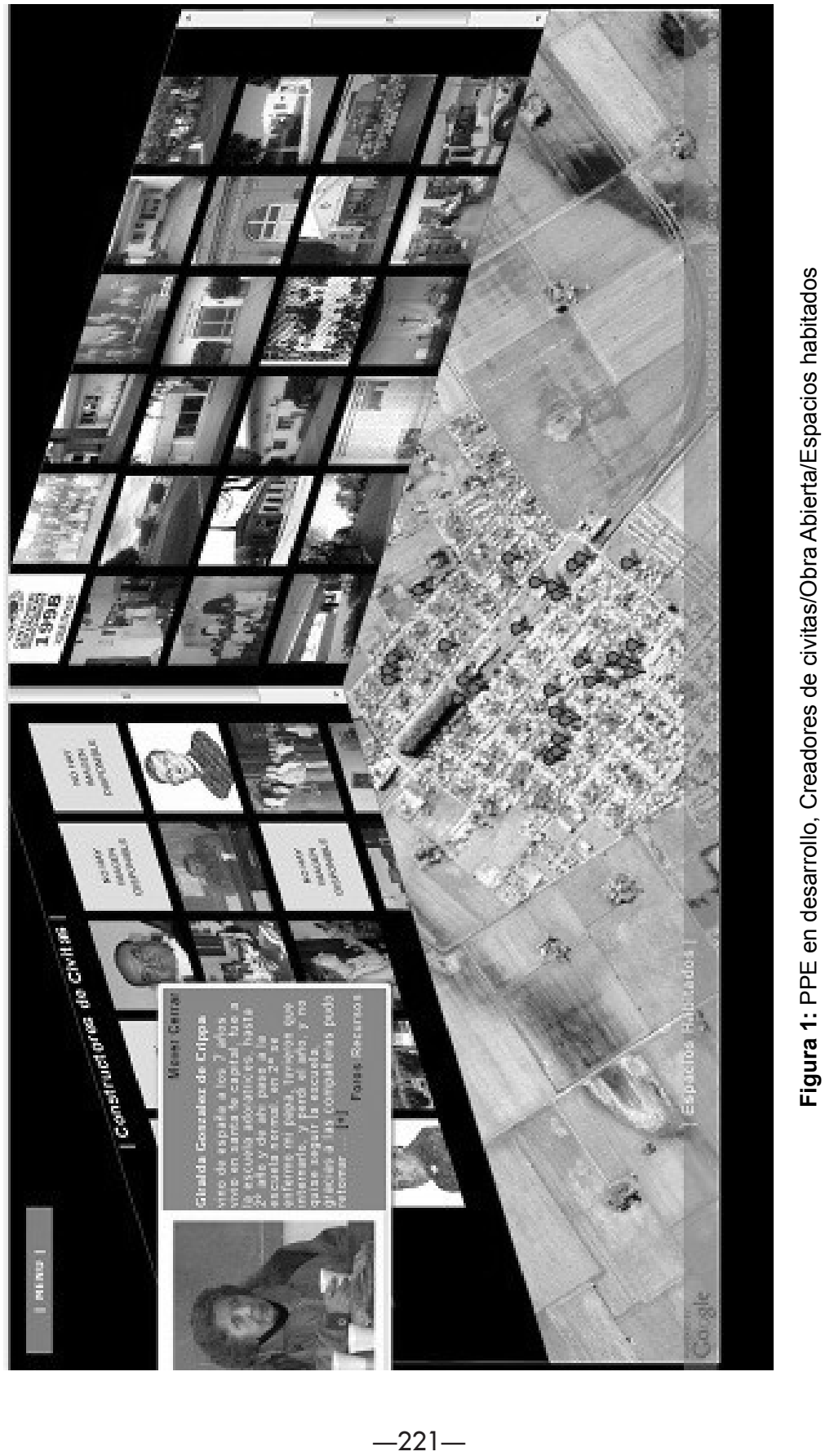


1) Exploración y lectura de contenidos (memorias y discusiones), (+)

2) Participación en responder encuestas, preguntas abiertas, adjuntar información en diversos formatos, comunicarse con otros en variados formatos, $\left(^{+}\right)$

3) Participación en espacios ad hoc con fines educativos, comunitarios, políticos, de producción, de recreación, etc.

De este modo, se propone la puesta en obra de los aspectos tecnológicos y sociales del DHD a través de espacios de navegación totalmente abierta y otros con mayores restricciones según grupos y responsabilidades distribuidas. A la vez es posible evaluar los porcentajes en las distintas instancias de participación dado que la sola exploración y lectura, resultaría insuficiente para los objetivos propuestos. La puesta en obra del DHD tendría que tender a un diálogo incremental entre los valores del nivel 2 y 3 que asegurarían el crecimiento escalar del sistema en base a los principios teóricos y metodológicos desarrollados en el presente texto.

\section{A modo de conclusión}

El proceso de reflexión y trabajo propio de la construcción de Telares de la Memoria en tanto primer prototipo de DHD, permite dar cuenta de los avances y reajustes propios de una propuesta de Investigación-Acción. La construcción "no a priori" del PPE permitió, el despliegue de un tiempo/espacio que en su mismo devenir experiencial se fue resignificando en los ritmos y presupuestos que como grupo de I+D (CIFASIS) y luego como grupo ampliado, se fueron gestando. El respeto a los tiempos del aprendizaje y semantización de los procesos que el proyecto fue abriendo, hoy se constituye en una de sus principales riquezas y habilita su continuidad.

Los procesos desencadenados en la discusión del qué, cómo y a quiénes en espacios democratizantes ampliados, han posibilitado el inicio de la puesta en juego de prácticas ciudadanas en torno a lo que podríamos llamar gobernanza, en tanto proceso de toma de decisiones que propone una horizontalidad en contraposición a las tradicionales relaciones y decisiones verticalistas. Sin embargo, aún resta abrir nuevos espacios, desandar los miedos, dar lugar a nuevos protagonistas, a otras narrativas de aquello que aparece como memoria individual pero que no deja de ser solo un punto de vista particular sobre la memoria colectiva que en definitiva condiciona el proceso de recuerdo individual, gobernando dinámicas de memoria, de olvido, remociones y reivindicaciones.

La escritura de "Telares...", no deja de interrogar sobre procesos conflictivos, en tanto se corre el riesgo de que algunos grupos queden excluidos de la participación. Esto pudo ser un obstáculo importante en relación a personas mayores a quienes se las convocaba como parte esencial del proyecto o para quiénes no disponen de tecnología y/o acceso a Internet. Situación que comienza a ser transformada a nivel local a partir de que los espacios públicos se comenzaron a rediseñar para provocar aprendizajes y accesos a las TIC. 
Valga como experiencia de integración, el trabajo generado a través del DHD entre adultos mayores (la mayoría de más de 80 años) y jóvenes de escuela media reunidos por un temática significativa donde se pusieron en juego nuevas relaciones de saber- poder y aprendizaje. Igualmente significativa, cabe mencionar la participación de ciudadanos que no disponían de medios materiales para el acceso a TIC en sus hogares.

Si bien la iniciativa de "Telares de la memoria" hoy se ubica en una localidad en particular, es intención que esta experiencia se pueda resignificar en otras realidades y en otros grupos, abriendo el debate sobre nociones acerca de los usos y apropiación de las TIC en procesos amplios de gobernanza. Se trata de habilitar una mirada hacia una realidad que excede los límites territoriales mostrando los límites que esta apropiación implica y la complejidad de su anclaje, donde queda claro que la sola posesión y acceso masivo a las TIC, no sostiene la revitalización de lo Público. (2)

\section{Notas Bibliográficas}

(1) Esta división tiene como expresión material el trazado de las vías del ferrocarril, las cuales permiten separar territorialmente al pueblo en dos: llamados actualmente Pueblo Nuevo y Pueblo Viejo.

(2) Para obtener mayor información sobre el trabajo consultar: http://dimensionesdhd. ifosisconicet.gov.ar

\section{Referencias}

- Borja, J. (2004). Reabrir Espacios Públicos. Políticas culturales y ciudadanía. México: UAM, Plaza y Valdés Editores.

- $\quad$ Bourdieu, P. (1999). Miseria del mundo. Buenos Aires: Fondo de Cultura Económica de Argentina.

- Candau, J. (2002). Antropología de la memoria. Buenos Aires: Nueva Visión.

- Crawford, A. (1998). Crime prevention and Community Safety: Politics, policies and practices. London: Longman.

- Cullen, C. (comp.) (2004). Filosofía, cultura y racionalidad crítica. Nuevos caminos para pensar la educación. Buenos Aires: Stella, La Crujía.

- $\quad$ Foucault, M. (1991). Saber y Verdad. Madrid: La Piqueta.

- García, R. (2007). Sistemas Complejos. Conceptos, método y fundamentación epistemológica de la investigación interdisciplinaria. Buenos Aires: Gedisa.

- Halbwachs, M. (1994). Les cadres sociaux de la memoire. Paris: Albin Michel.

- Hammersley, M. y Atkinson P. (1994). Etnografía. Métodos de investigación. Barcelona: Paidós.

- Laddaga, R. (2010). Estética de la emergencia. Buenos Aires: Adriana Hidalgo editora.

- Lea J. y Young, J. (2001). Que hacer con la ley y el orden. Buenos Aires: Editores del Puerto.

- $\quad$ Nora, P. (1997). Les lieux de Mémoire. París: Gallimard.

- Reguillo, R. (2005). Sociabilidad, inseguridad y miedos. Una trilogía para pensar la ciudad contemporánea. Buenos Aires: CIE-UNSAM. 
- $\quad$ San Martín, P. (2003). Hipertexto: Seis propuestas para este milenio. Buenos Aires: La Crujía.

- $\quad$ San Martín, P. y Guarnieri, G. (2009). Construir un dispositivo hipermedial dinámico en la Universidad Pública. Cuadernos Sociales, 9 (1), 111-127.

- $\quad$ San Martín, P.; Guarnieri, G; Rodriguez, G; Bongiovani, P. y Sartorio, A. (2010). El dispositivo Hipermedial Dinámico Campus Virtual UNR. Extraído el 3 de abril de 2010 desde http://rephip.unr.edu.ar/handle/2133/1390

- $\quad$ San Martín, P.; Sartorio, A.; Guarnieri, G. y Rodríguez, G. (2008). Hacia un dispositivo hipermedial dinámico: Educación e investigación para el campo audiovisual interactivo. Buenos Aires: Universidad Nacional de Quilmes.

- $\quad$ Thomas, H.; Buch, A. (compiladores) (2008). Actos, actores y artefactos. Buenos Aires: Universidad Nacional de Quilmes.

- $\quad$ Tosello M.E.; Brendanini, M.G.; Mines, P. y Carrara, L. (2010). Imaginando el Espacio del Conocimiento. Interfaz experimental para una Biblioteca virtual de Arquitectura y Diseño. Santa Fe: Universidad Nacional del Litoral.

- Violi, P. (2010) Recordar el futuro. Museos de la memoria e identidades culturales. En Dalmaso, M.T.; Andacht, F; Fatala, N (comp.) (2010) Tiempo, espacio e identidades. Buenos Aires: La Crujía.

- Young, J. (2003). La sociedad excluyente. Exclusión social, delito y diferencia en la Modernidad tardía. Barcelona: Marcial Pons.

\section{Agradecimientos}

A todos los integrantes de "Telares de la memoria" y especialmente a Finita Burzio, colaboradora incondicional de Wheelwright. Este proyecto de I+D se lleva adelante con el apoyo de los subsidios INNOVA 2009 de la Secretaría de Estado de Ciencia, Tecnología en Innovación de la Provincia de Santa Fe y Subsidio PIP del CONICET (2009-2011) N 0718 Proyecto "Obra Abierta: DHD para educar e investigar". 